\title{
Nanoscale Investigation of Composition and Grain Boundary Effects in Magnetic Hard Disk Media
}

\author{
Juliet Risner, Unoh Kwon and Robert Sinclair
}

Stanford University, Department of Materials Science and Engineering, Stanford, CA 94305-2205

Longitudinal recording media have shown remarkable longevity in the fast-paced computer hardware industry. While a variety of factors have contributed to the continued dominance of such magnetic hard disks, the role of atomic segregation to the grain boundaries has proven tantamount to their success [1]. Further decreasing grain size past the approximately $10 \mathrm{~nm}$ in current media would hasten the detrimental effects of thermal fluctuations. Therefore, the ability to shield neighboring grains by segregation of nonmagnetic species becomes of heightened interest. High resolution transmission electron microscopy (TEM) and nanoprobe $\mathrm{x}$-ray energy dispersive spectroscopy (EDS) is clearly an important technique in characterizing these small-scale microstructures.

Fig. 1 is a high resolution image of grains in longitudinal $\mathrm{CoCrX}$ media with nanoprobe positions specified. Fig. 2 shows the results of EDS analysis on samples where $\mathrm{X}=\mathrm{Ta}$, $\mathrm{Pt}$, or $\mathrm{TaPt}$, respectively. As reported previously, the addition of Ta enhances segregation, while Pt has only a slight influence $[2,3]$. The addition of equal amounts of both species (Fig. 2c) has an intermediate effect. Further experimentation intends to test, using high resolution TEM and nanoprobe EDS, whether grain boundary orientations exhibiting high atomic coincidence have less $\mathrm{Cr}$ segregation than those with minimal atomic coincidence. Prior research by Wittig et al. suggested a link between the grain boundary orientation in $\mathrm{CoCrX}$ longitudinal media and the degree of $\mathrm{Cr}$ segregation [4].

Additionally, there is similar interest in the grain boundary structure of perpendicular media. Thicker intergranular regions and smaller grain size have been observed when oxygen replaces Ta or $\mathrm{B}$ in the magnetic layer in perpendicular media [5]. These changes give rise to the further increase in recording density and the improvement of signal-to-noise ratio.

Fig. 3 is a bright field image of grain boundary structures with selected area diffraction patterns (SADP) of boron and oxygen-containing perpendicular media, respectively. The SADPs show that both samples have preferred vertical c-axis grain alignment. The CoCrPtO alloy, however, has thicker boundary regions and smaller grain size than the CoCrPtB alloy. Fig. 4 shows the grain size analysis for these samples in the form of a cumulative percentage plot. The higher slope and smaller standard deviation of the CoCrPtO curve represent a more uniform grain size distribution than the CoCrPtB. The well-isolated grain structures separated by a non-magnetic oxide phase in the $\mathrm{CoCrPtO}$ alloy play an important role in the exchange decoupling of Co alloy grains.

\section{References}

[1] J.E. Wittig, et al., IEEE Trans. Magn. 34 (1998) 1564.

[2] J.E. Wittig et al., Mat. Res. Soc. Symp. Proc. 517 (1998) 211.

[3] R. Sinclair et al., Proc. Microsc. Microanal. 8 (suppl. 2) (2002) 368. 
[4] J.E. Wittig et al., Proc. Microsc. Microanal. 7 (2001) 298.

[5] S. Oikawa et al., IEEE Trans. Magn. 36 (2000) 2393.

[6] This work was supported by Komag Corporation and one author (JR) acknowledges the receipt of a NSF Graduate Fellowship.
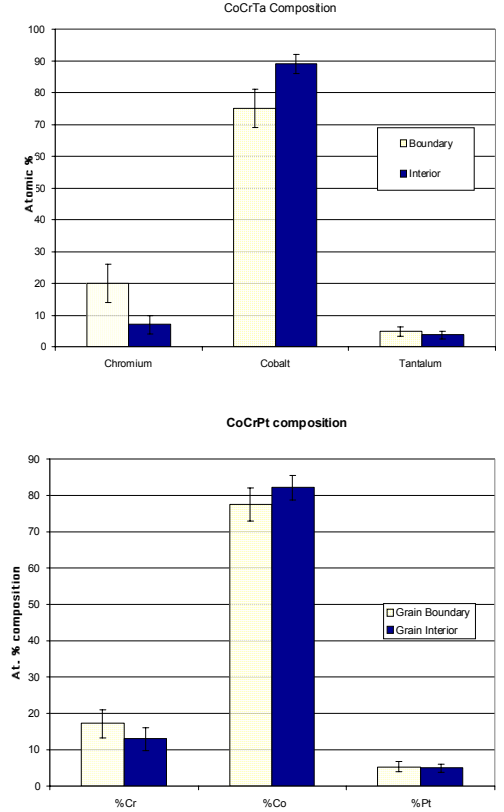

CoCrPtTa Composition
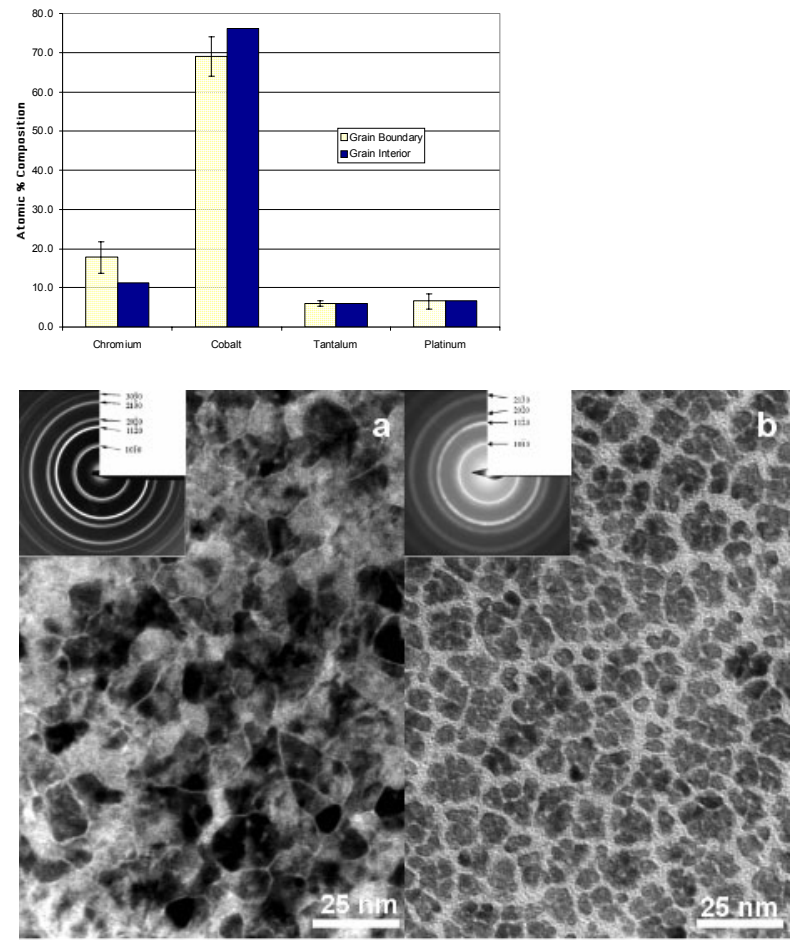

Fig. 3. Bright field images and selected area diffraction patterns of (a) CoCrPtB and (b) CoCrPtO media.
Fig. 1. Grain boundary vs. grain interior composition charts for $\mathrm{CoCrX}$ longitudinal media. (a) $\mathrm{X}=\mathrm{Ta}$ (b) $\mathrm{X}=\mathrm{Pt}$ (c) $\mathrm{X}=\mathrm{TaPt}$.

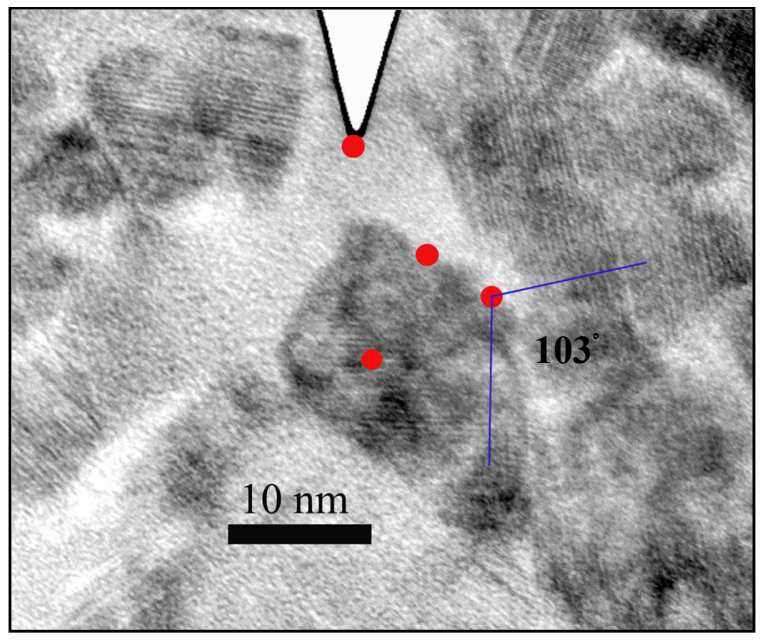

Fig. 2. High resolution image of $\mathrm{CoCrX}$ longitudinal media showing nanoprobe positions similar to those used to generate the data in Fig. 1.

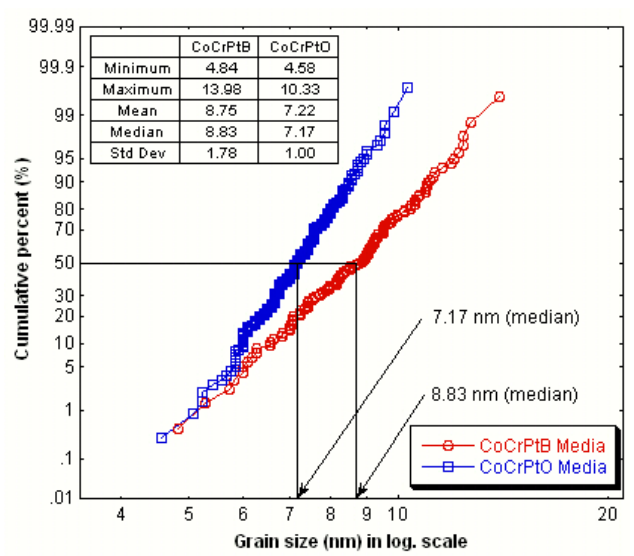

Fig. 4. Grain size analysis of CoCrPtB and CoCrPtO alloy in the form of a cumulative percentage plot. 